\title{
Study on the Drug-Drug Interaction of Antituberculosis Drugs — PA-824 and Moxifloxacin Based on Pharmacokinetics in Rats by LC-MS/MS
}

\author{
Juan Jing ${ }^{1,2}$, Yuting $\mathrm{Jia}^{2}$, Tian Feng ${ }^{2}$, Tian $\mathrm{Xie}^{1}$, Zhoupeng $\mathrm{Li}^{1}$, Yong $\mathrm{Hao}^{1 *}$ and Libin Wang ${ }^{2 *}$ \\ ${ }^{1}$ NCO School, Army Medical University, Shijiazhuang 050081, China \\ ${ }^{2}$ Department of Medicinal Chemistry, School of Pharmacy, The Fourth Military Medical University, Xi'an 710032, China
}

Received: 07 February 2018; accepted: 14 March 2018

\begin{abstract}
A simple, rapid, and sensitive liquid chromatography-tandem mass spectrometry (LC-MS/MS) method was developed and validated for the simultaneous quantitation of PA- 824 and moxifloxacin in rat plasma using carbamazepine as an internal standard (IS). The sample preparation involved a one-step protein precipitation method with methanol. The separation was performed on Inertsi ${ }^{\circledR}$ ODS3 C18 column $(150 \mathrm{~mm} \times 4.6 \mathrm{~mm}, 5 \mu \mathrm{m})$ and maintained at $30{ }^{\circ} \mathrm{C}$. The mobile phase consisted of $0.1 \%$ formic acid in acetonitrile-water $(90: 10 \mathrm{v} / \mathrm{v})$ with fast isocratic elution at a flow rate of $0.6 \mathrm{~mL} / \mathrm{min}$ and a run time of $10 \mathrm{~min}$. A mass spectrometer was run in the positive ion electrospray ionization (ESI) mode using multiple reaction monitoring (MRM) to monitor the mass transitions. The MRM transitions were chosen to be $m / z 360.1 \rightarrow m / z 175.0$ for PA-824, $m / z 402.0 \rightarrow m / z 383.9$ for moxifloxacin, and $m / z 237.1 \rightarrow m / z 194.0$ for IS. The method was fully validated in terms of selectivity, linearity, accuracy, precision, matrix effect, recovery, and stability, respectively. The method was successfully applied to drug-drug interaction (DDI) study of PA-824 and moxifloxacin in rats. The results show that the main pharmacokinetic parameters of PA-824, namely, $T_{\max }, t_{1 / 2}$, and $\mathrm{AUC}_{(0-t)}$, increased more in the PA-824 and moxifloxacin group than in the PA-824 group. However, there were little changes in the main pharmacokinetic parameters of moxifloxacin from single and combined groups.
\end{abstract}

Keywords: LC-MS/MS, drug-drug interaction, PA-824, moxifloxacin

\section{Introduction}

Tuberculosis (TB) is an infectious disease of international importance and ranks among the top 10 causes of death in the world [1]. It is estimated that over 8 million people contract tuberculosis each year, and approximately 2 to 3 million people die of this disease $[2,3]$. These situations, particularly the global resurgence of $\mathrm{TB}$ and the rapid emergence of multi-drug-resistant TB (MDR-TB), underscore the importance of the development of new anti-tuberculosis drugs and new protocols for efficacious clinical control of TB patients using ordinary anti-mycobacterial drugs [4]. Unfortunately, no new drugs, except rifabutin and rifapentine, have been marketed for TB in the US and other countries during the 40 years after the release of rifampicin [5]. There are a number of constraints that have deterred companies from investing in new anti-TB drugs. PA-824, a new chemical entity and a member of a class of compounds known as nitroimidazo-oxazines, has significant anti-tuberculosis activity and a unique mechanism of action [6]. TB treatment regimen for both drug-sensitive and MDR-TB was investigated in a Phase II proof-of-concept clinical trial [7, 8]. The NC-001 (J-M-Pa-Z) study was carried out in treatment-naive, drug-susceptible patients with uncomplicated pulmonary $\mathrm{TB}$ at two sites in Cape Town, South Africa, between October 2010 and August 2011 [8]. In this research, the PaMZ (PA-824, moxifloxacin, and pyrazinamide) combination has the most rapid onset in early bactericidal activity (EBA) measured with the decrease in the logarithm of colony-forming units (log CFU [EBACFU]) over time with a marginal deceleration in activity at 8.5 days. The PaMZ regimen might be a potential treatment-shortening regimen, but the interaction between the three drugs is not clear. Will there be drug-drug interaction (DDI) between the

*Authors for correspondence: yonghao12000@aliyun.com,wtpierce@163.com three drugs? What will be the impact on the clinical application of PaMZ by DDI? In our previous study [9], a liquid chromatography-tandem mass spectrometry (LC-MS/MS) method has been developed and validated for simultaneous determination of PA-824, moxifloxacin, and pyrazinamide in rat plasma and its application to a pharmacokinetic study in rats. To further clarify the DDI between the three drugs, in the present study, we investigated and compared the pharmacokinetics of coadministration of the two drugs (PA-824 and moxifloxacin, Figure 1) in rats. Despite PA-824 and moxifloxacin having good pharmacological activity, combination therapy with the two drugs has not been reported; also, the interaction between the two drugs is not clear. A number of studies reported the development of analytical methods for quantitation of PA-824 or moxifloxacin alone [10-14]. However, there are no study to provide information about the simultaneous determination of PA-824 and moxifloxacin using LC-MS/MS and the pharmacokinetic interaction between PA-824 and moxifloxacin. The aim of this article was to develop and validate a simple, specific, and sensitive LC-MS/MS method for simultaneous determination of PA-824 and moxifloxacin in rat plasma and to apply this method to a pharmacokinetic study to evaluate the drug-drug interactions between the two drugs. Significant differences in pharmacokinetic properties were observed between the single and combined groups after administration of equal dose of PA-824 and moxifloxacin.

\section{Materials and Methods}

Materials and Reagents. Moxifloxacin and carbamazepine were purchased from Sigma-Aldrich (St. Louis, MO, USA). PA-824 was a kind gift from Prof. Shengyong Zhang (Department of Medicinal Chemistry, The Fourth Military Medical University). Chromatographic grade acetonitrile or

This is an open-access article distributed under the terms of the Creative Commons Attribution-NonCommercial 4.0 International License (https://creativecommons.org/licenses/by-nc/4.0/), which permits unrestricted use, distribution, and reproduction in any medium for non-commercial purposes, provided the original author and source are credited, a link to the CC License is provided, and changes - if any - are indicated. 
$\mathrm{O}_{2} \mathrm{~N}$

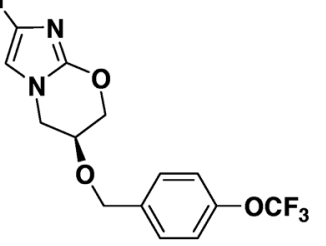

A

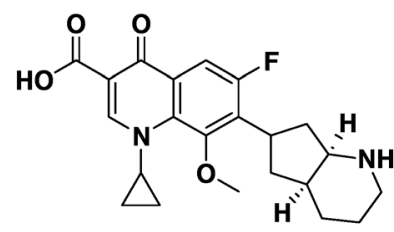

B

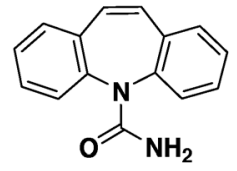

C

Figure 1. Chemical structures of PA-824 (A), moxifloxacin (B), and internal standard carbamazepine $(\mathrm{C})$

methanol (Dikma, Richmond Hill, New York, USA) and analytical grade formic acid (Tianjin Fuyu Chemical, Tianjin, China) were used for the preparation of the mobile phase. Ultrapure water used in the experiments was prepared via a Milli-Q water purification system (Millipore, Bedford, MA, USA). Other solvents and reagents were of analytical grade.

Animals, Drug Administration, and Sampling. Sprague Dawley rats $(200 \pm 20 \mathrm{~g})$ were purchased from the Experimental Animal Center of the Fourth Military Medical University (Shaanxi, China). All the studies on animals were in accordance with the Guidelines for the Care and Use of Laboratory Animals. The rats were acclimated in the laboratory for 1 week prior to the experiments, housed in separate cages at a temperature of $23 \pm 2{ }^{\circ} \mathrm{C}$ with a $12 \mathrm{~h}$ light-dark cycle and a relative humidity of $50 \%$, and with free access to standard water and diet. The rats were fasted for $12 \mathrm{~h}$ before oral administration with free access to water.

The drugs were dissolved in $0.5 \%$ CMC-Na before oral administration. In the pharmacokinetic study, 24 rats were randomly divided into three groups ( $n=8$ per group), and PA-824 (20 mg/mL), moxifloxacin $(40 \mathrm{mg} / \mathrm{mL})$, and PA-824 $(20 \mathrm{mg} / \mathrm{mL})$ and moxifloxacin $(40 \mathrm{mg} / \mathrm{mL})$ were orally administered to each group, respectively. Blood samples $(0.3 \mathrm{~mL})$ were collected by retro-orbital puncture at $5,10,15,30,60,120,240$, $360,480,720,1440$, and 2160 min post-dosing after the drugs were orally administered to the rats. Then, each blood sample was immediately centrifuged at approximately $12,000 \mathrm{rpm}$ and $4{ }^{\circ} \mathrm{C}$ for $15 \mathrm{~min}$, and a $100 \mu \mathrm{L}$ aliquot of supernatant plasma layer was transferred into another tube and stored at $-80{ }^{\circ} \mathrm{C}$ until analysis. An aliquot of $100 \mu \mathrm{L}$ rat plasma sample was mixed with $10 \mu \mathrm{L}$ of internal standard (IS) solution (100 ng/mL), and $800 \mu \mathrm{L}$ methanol was added. The sample was vortex-mixed for $3 \mathrm{~min}$, and the mixture was separated by centrifugation at $12,000 \mathrm{rpm}$ for $15 \mathrm{~min}$. An aliquot of $500 \mu \mathrm{L}$ of the protein-free supernatant was then evaporated to dryness at $40{ }^{\circ} \mathrm{C}$ under nitrogen. The resulting extract was dissolved in $50 \mu \mathrm{L}$ of methanol and vortex-mixed for $3 \mathrm{~min}$. After centrifugation at $12,000 \mathrm{rpm}$ for $15 \mathrm{~min}$, an aliquot of $5 \mu \mathrm{L}$ supernatant was injected into the LC-MS/MS.

Preparation of Stock Solutions. Standard stock solutions of all compounds were prepared in methanol at $1 \mathrm{mg} / \mathrm{mL}$. Then, the stock solutions were diluted with methanol to obtain fresh standard working solution. All the solutions were stored at $-20{ }^{\circ} \mathrm{C}$ and were brought to room temperature before use.

LC-MS/MS Analysis. The liquid chromatography was performed using an Agilent 1260 Series liquid chromatograph (Agilent Technologies, Palo Alto, CA, USA), which included an Agilent 1260 Quat pump VL, an Agilent 1260 autosampler, and a temperature-controlled column compartment; chromatographic separation was achieved on an Inertsil ${ }^{\circledR}$ ODS3 C18 column $(150 \mathrm{~mm} \times 4.6 \mathrm{~mm}, 5 \mu \mathrm{m})$ and maintained at $30^{\circ} \mathrm{C}$. The mobile phase consisted of $0.1 \%$ formic acid in acetonitrile-water $(90: 10 \mathrm{v} / \mathrm{v})$ with fast isocratic elution at a flow rate of $0.6 \mathrm{~mL} / \mathrm{min}$ and a run time of $10 \mathrm{~min}$. The sample volume injected was $5 \mu \mathrm{L}$ and the temperature of the autosampler was set at $4{ }^{\circ} \mathrm{C}$. The LC system was coupled with an Agilent 6460 triple quadrupole mass spectrometer (USA) equipped with an electrospray ionization source. The mass spectrometer was run in the positive ion electrospray ionization (ESI) mode using multiple-reaction monitoring (MRM) to monitor the mass transitions. The MRM transitions were chosen to be $m / z 360.1 \rightarrow m / z 175.0$ for PA-824, $m / z 402.0 \rightarrow m / z 383.9$ for moxifloxacin, and $\mathrm{m} / \mathrm{z} 237.1 \rightarrow \mathrm{m} / \mathrm{z} 194.0$ for IS. The fragmentor voltage values for PA-824, moxifloxacin, and IS were $65 \mathrm{~V}, 135 \mathrm{~V}$, and $120 \mathrm{~V}$, respectively. The collision energy values for PA-824, moxifloxacin, and IS were $35 \mathrm{eV}, 20 \mathrm{eV}$, and $20 \mathrm{eV}$, respectively.

Method Validation. The method was validated according to the Food and Drug Administration (FDA) Guidelines for Biological Method Validation [15].

Selectivity. The selectivity was assessed by comparing blank plasma samples obtained from six rats with those of the corresponding spiked samples or with the actual samples to conform the presence or absence of interference.

Linearity and Lower Limit of Quantification (LLOQ). Linearity was analyzed by the weighted regression method $\left(1 / x^{2}\right)$ of peak area ratios of PA-824 and moxifloxacin to IS versus actual concentrations. The calibration curves $(n=7)$ were prepared by spiking blank plasma with standard solution of PA-824, moxifloxacin, and IS. Final concentrations of the calibration standards were $5,50,100,500,1000,2000$, and $5000 \mathrm{ng} / \mathrm{mL}$ in rat plasma. LLOQ was determined in accordance to the base line noise, considering a signal-to-noise ratio of 10:1.

Precision and Accuracy. Accuracy and precision were evaluated in six replicates at three different quality control (QC) levels on the same day and for three consecutive days using the calibration curve, respectively. The accuracy of all validation tests was expressed as the relative error ( $\mathrm{RE} \%$ ) obtained by calculating the percentage difference between the found concentration over that of the spiked value. The precision was denoted by the relative standard deviation (RSD\%). The accuracy should not exceed $15 \%$, and the precision was required to be within $\pm 15 \%$ for all levels, except $\pm 20 \%$ for the LLOQ, which was defined to be the lowest concentration that could be quantified.

Recovery and Matrix Effects. The extraction recoveries were determined by comparing the mean peak areas of QC samples (six replicates at lower QC, middle QC, and higher QC levels) with the mean peak areas of the spike-after-extraction samples at the same concentrations. The matrix effects were evaluated as the ratio of the peak areas of samples spiked postextraction to those in non-extracted samples at equivalent concentrations $(n=6)$.

Stability. The stability of analyte in rat plasma was investigated by analyzing five replicates at three QC levels for short-term storage (room temperature for $12 \mathrm{~h}$ ), long-term storage $\left(-20{ }^{\circ} \mathrm{C}\right.$ for 14 days), and freeze-thaw cycles. Postpreparation stability was assessed by analyzing the extracted samples kept in the autosampler at $4{ }^{\circ} \mathrm{C}$ for $24 \mathrm{~h}$. The acceptance criteria were set from $85 \%$ to $115 \%$.

Pharmacokinetic and Statistical Analysis. All data were subsequently processed by the Agilent MassHunter software and 
Statistics version 2.0 (DAS 2.0, Anhui Provincial Center for Drug Clinical Evaluation). Terminal elimination rate constant $(\mathrm{Ke})$ was determined by linear regression of the terminal portion of plasma concentration-time data, and the elimination half-life $\left(t_{1 / 2}\right)$ was calculated to be $0.693 / \mathrm{Ke}$. The area under the plasma concentration curve (AUC) versus time $\left(\mathrm{AUC}_{0-t}\right)$ from time 0 to the time of the last measured concentration $\left(C_{\text {last }}\right)$ was calculated using the linear trapezoidal rule. The AUC from time 0 to infinity $\left(\mathrm{AUC}_{0-\infty}\right)$ was obtained by the addition of $\mathrm{AUC}_{0-t}$ and the extrapolated area determined by Clast/Ke. Data were shown as mean \pm standard deviation (mean \pm SD). Statistical analysis was computed using SPSS 13.0 software. All the data are expressed as mean $\pm \mathrm{SD}$, and a $p$ value $<0.05$ was deemed to be statistically significant.

\section{Results and Discussion}

Method Development and Optimization. It was found that direct protein precipitation with typically employed precipitating agents produced low recovery with high background noise. Thus, we have chosen liquid-liquid extraction to carry out the quantitative analysis. The ion abundances of PA-824, moxifloxacin, and IS were significantly greater in the positive-ion mode than in the negative-ion mode. Additionally, the MRM transitions were chosen to be $\mathrm{m} / \mathrm{z} 360.1 \rightarrow \mathrm{m} / \mathrm{z} 175.1$ for PA-824, $m / z 402.0 \rightarrow m / z 383.9$ for moxifloxacin, and $m / z$ $237.1 \rightarrow m / z 194.0$ for IS. Acetonitrile was selected as the organic portion of the mobile phase, and it was found that the addition of $0.1 \%$ formic acid in the organic phase could enhance the sensitivity and improve the peak shapes of PA-824, moxifloxacin, and IS. Then, different volume ratios of acetonitrile were tested as the organic portion of the mobile phase, and finally, acetonitrile and water in a volume ratio of 9:1 by isocratic elution produced the best separating effect and the shortest run time. Several attempts were made with different C18 HPLC columns, such as Agilent Eclipse plus C18 column $(100 \mathrm{~mm} \times 2.1 \mathrm{~mm}, 3.5 \mu \mathrm{m})$, Waters Acquity BEH C18 column $(50 \mathrm{~mm} \times 2.1 \mathrm{~mm}, 1.7 \mu \mathrm{m})$, and Zorbax SB-C18 column $(150 \mathrm{~mm} \times 2.1 \mathrm{~mm}, 5 \mu \mathrm{m})$; the best chromatographic separation and response were achieved on Inertsil ${ }^{\circledR}$ ODS3 C18 column $(150 \mathrm{~mm} \times 4.6 \mathrm{~mm}, 5 \mu \mathrm{m})$. The IS (carbamazepine) showed good peak characteristics with high response, well separated from both the analytes and free from interference with detection of analytes. On this column PA-824, moxifloxacin, and IS were well separated from the potential endogenous interferential substances and showed suitable retention without compromising separation efficiency and peak shape.

\section{Method Validation}

Selectivity. The selectivity of the method was assessed by LC-MS/MS derived from blank biological samples. Figure 2 shows the typical chromatograms of a blank plasma sample; a blank plasma sample spiked with PA-824, moxifloxacin, and IS; and a plasma sample from a rat, 15 min after an oral administration of PA-824, moxifloxacin, and IS. The result demonstrated that there was no interference with the determination of the PA-824, moxifloxacin, and IS.

Linearity and LLOQ. The standard calibration curve for spiked rat plasma containing PA-824 was linear over the range $5-5500 \mathrm{ng} / \mathrm{mL}$ with a correlation coefficient $r>0.9978$. In addition, the standard calibration curve for spiked rat plasma containing moxifloxacin was linear over the range of 5-5500 ng/mL with a correlation coefficient $r>0.9961$. The LLOQ was $5 \mathrm{ng} / \mathrm{mL}$ for PA-824 and moxifloxacin, which was sensitive and accurate enough for monitoring the pharmacokinetics of the drugs. The results indicated that the calibration curve was linear, accurate, and precise over the range of the method.

Precision and Accuracy. The intra-day and inter-day precision and accuracy were shown in Table 1 . The intra-day and inter-day RSDs (\%) were both not more than $7.9 \%$, while the $\mathrm{RE}(\%)$ of assay accuracies ranged from $-5.4 \%$ to $8.9 \%$. These results suggest that the method was accurate and precise over the range of the assay.

Table 1. Precision and accuracy of PA-824 and moxifloxacin in rat plasma

\begin{tabular}{lcccccc}
\hline Compound & $\begin{array}{c}\text { Concentration } \\
(\mathrm{ng} / \mathrm{mL})\end{array}$ & \multicolumn{2}{c}{ Intra-day $(n=6)$} & & \multicolumn{2}{c}{ Inter-day $(n=6 \times 3)$} \\
\cline { 7 - 7 } \cline { 6 - 7 } & & $\begin{array}{c}\text { Precision } \\
(\mathrm{RSD} \%)\end{array}$ & $\begin{array}{c}\text { Accuracy } \\
(\mathrm{RE} \%)\end{array}$ & & $\begin{array}{c}\text { Precision } \\
(\mathrm{RSD} \%)\end{array}$ & $\begin{array}{c}\text { Accuracy } \\
(\mathrm{RE} \%)\end{array}$ \\
\hline PA-824 & 20 & 4.3 & 6.2 & & 4.8 & 5.6 \\
& 500 & 5.4 & 2.7 & & 7.2 & -2.6 \\
& 5000 & 4.9 & -2.3 & & 3.1 & 3.1 \\
Moxifloxacin & 20 & 7.9 & 2.8 & & 5.2 & 4.1 \\
& 500 & 6.7 & 5.4 & & 6.7 & 8.9 \\
& 5000 & 7.4 & 8.7 & & 2.9 & -5.4 \\
\hline
\end{tabular}

(1)

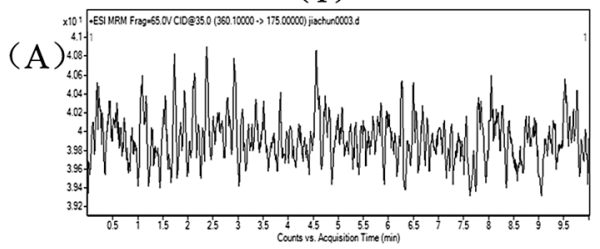

(B)
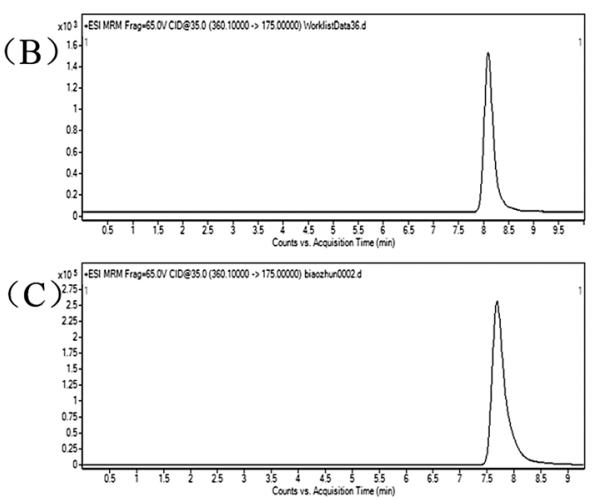

(2)
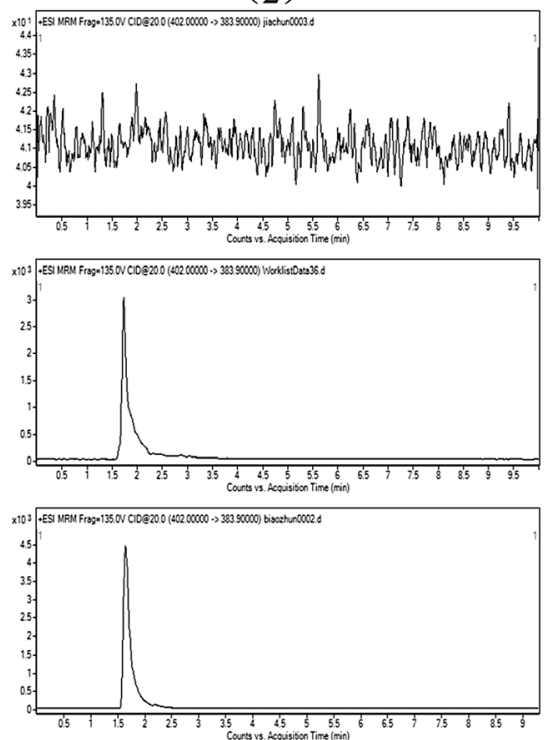

(3)
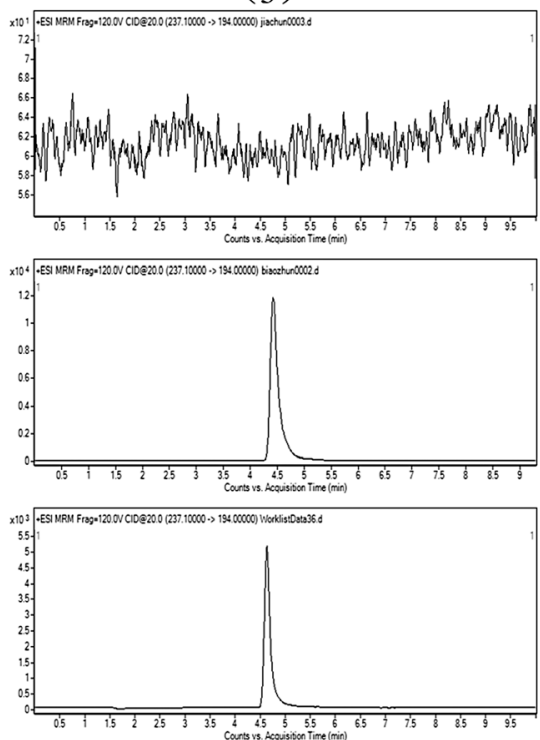

Figure 2. Typical MRM chromatograms of PA-824 (1), moxifloxacin (2), and IS (3) in rat plasma: (a) blank rat plasma; (b) blank rat plasma spiked with PA-824 (10 ng/mL), moxifloxacin $(10 \mathrm{ng} / \mathrm{mL})$, and IS $(100 \mathrm{ng} / \mathrm{mL})$; and (c) a plasma sample obtained $15 \mathrm{~min}$ after oral administration of PA-824 $(20 \mathrm{mg} / \mathrm{kg})$ and moxifloxacin $(40 \mathrm{mg} / \mathrm{kg})$ in rats 
Recovery and Matrix Effects. The mean extraction recoveries of the investigated components in plasma at three different concentration levels were found to be $84.32-98.21 \%$ with RSD of less than $5.7 \%$. Matrix effects on the recovery of blank plasma samples spiked after the sample preparation with low, medium, and high concentration levels of all three analytes were found to be within the acceptable limits. The same evaluation was performed on the IS $(100 \mathrm{ng} / \mathrm{mL})$, and no significant peak area differences were detected. Thus, it was demonstrated that the plasma matrix effect was negligible for the assay. The results were shown in Table 2.

Table 2. Matrix effect for PA-824, moxifloxacin, and IS in rat plasma

\begin{tabular}{|c|c|c|c|c|c|c|c|}
\hline \multirow{2}{*}{$\begin{array}{l}\text { Concentration } \\
(\mathrm{ng} / \mathrm{mL})\end{array}$} & \multicolumn{3}{|c|}{ PA-824 } & \multicolumn{3}{|c|}{ Moxifloxacin } & \multirow{2}{*}{$\frac{\text { IS }}{1000}$} \\
\hline & 20 & 500 & 5000 & 20 & 500 & 5000 & \\
\hline \multirow[t]{5}{*}{ Recovery (\%) } & 86.3 & 90.4 & 102.3 & 94.1 & 97.8 & 105.6 & 98. \\
\hline & 93.8 & 87.6 & 114.3 & 99.6 & 98.5 & 113.1 & \\
\hline & 89.1 & 97.4 & 98.4 & 87.4 & 89.3 & 99.1 & 92 \\
\hline & 93.2 & 94.3 & 108.1 & 95.4 & 94.5 & 105.1 & 96 \\
\hline & 95.4 & 88.3 & 99.3 & 97.5 & 93.1 & 100.2 & 99. \\
\hline$n$ & 5 & 5 & 5 & 5 & 5 & 5 & 5 \\
\hline Mean (\%) & 92.1 & 91.6 & 104.4 & 94.8 & 94.6 & 104.6 & 96 \\
\hline SD & 3.7 & 4.2 & 6.7 & 4.6 & 3.7 & 5.5 & 3. \\
\hline RSD (\%) & 4.1 & 4.5 & 6.3 & 4.8 & 3.9 & 5.3 & 3.2 \\
\hline
\end{tabular}

Stability. As shown in Table 3, the rat plasma samples were stable at $-20{ }^{\circ} \mathrm{C}$ for at least 4 weeks with no significant loss. Plasma samples were stable over at least three freezethaw cycles.

Pharmacokinetic Application. The LC-MS/MS method was applied to the determination of PA-824, moxifloxacin, and their combination in rat plasma; the method has been successfully applied to pharmacokinetic interaction study of PA-824 and moxifloxacin in rats. In this study, we compared the pharmacokinetic parameters of rats, where PA-824, moxifloxacin, and their combination were administered. Mean plasma concentration-time curves $(n=8)$ were presented in Figure 3. The main pharmacokinetic parameters calculated using non-compartmental analysis were shown in Table 4. For PA-824, the coadministration of PA-824 and moxifloxacin caused a significant increase in the $T_{\max }$ and $\mathrm{AUC}_{(0-t)}$, and the coadministration of PA-824 and moxifloxacin caused little changes in the other main pharmacokinetic parameters. For moxifloxacin, the coadministration of moxifloxacin and PA-824 caused little changes in the main pharmacokinetic parameters. The results show that pharmacokinetic interactions among the drugs used in the treatment of TB are obviously indispensable.

Table 3. Determination of the stability of PA-824 and moxifloxacin

\begin{tabular}{lccccc}
\hline Compound & $\begin{array}{c}\text { Concentration } \\
(\mathrm{ng} / \mathrm{mL})\end{array}$ & $\begin{array}{c}\text { Frozen for 30 days } \\
(\mathrm{m} m \pm \mathrm{SD}, \%)\end{array}$ & $\begin{array}{c}\text { Three freeze-thaw cycles } \\
(\text { mean } \pm \mathrm{SD}, \%)\end{array}$ & $\begin{array}{c}\text { Room temperature for 12 } \mathrm{h} \\
(\text { mean } \pm \mathrm{SD}, \%)\end{array}$ & $\begin{array}{c}\text { Prepared samples in freezer }\left(-20{ }^{\circ} \mathrm{C}\right) \\
\text { for 24 } \mathrm{h}(\mathrm{mean} \pm \mathrm{SD}, \%)\end{array}$ \\
\hline PA-824 & 20 & $106.5 \pm 6.0$ & $105.0 \pm 8.0$ & $100.0 \pm 1.5$ & $102.5 \pm 4.0$ \\
& 500 & $103.7 \pm 8.5$ & $107.3 \pm 11.3$ & $112.3 \pm 8.5$ & $108.8 \pm 10.7$ \\
Moxifloxacin & 5000 & $96.6 \pm 3.5$ & $104.9 \pm 3.4$ & $98.9 \pm 3.6$ & $96.3 \pm 2.9$ \\
& 20 & $118.5 \pm 11.5$ & $98.0 \pm 11.0$ & $105.5 \pm 6.0$ & $111.0 \pm 11.0$ \\
& 500 & $97.9 \pm 7.4$ & $104.2 \pm 15.7$ & $99.5 \pm 5.4$ & $103.8 \pm 14.4$ \\
& 5000 & $100.4 \pm 5.7$ & $103.9 \pm 4.7$ & $97.4 \pm 4.9$ & $100.9 \pm 6.5$ \\
\hline
\end{tabular}
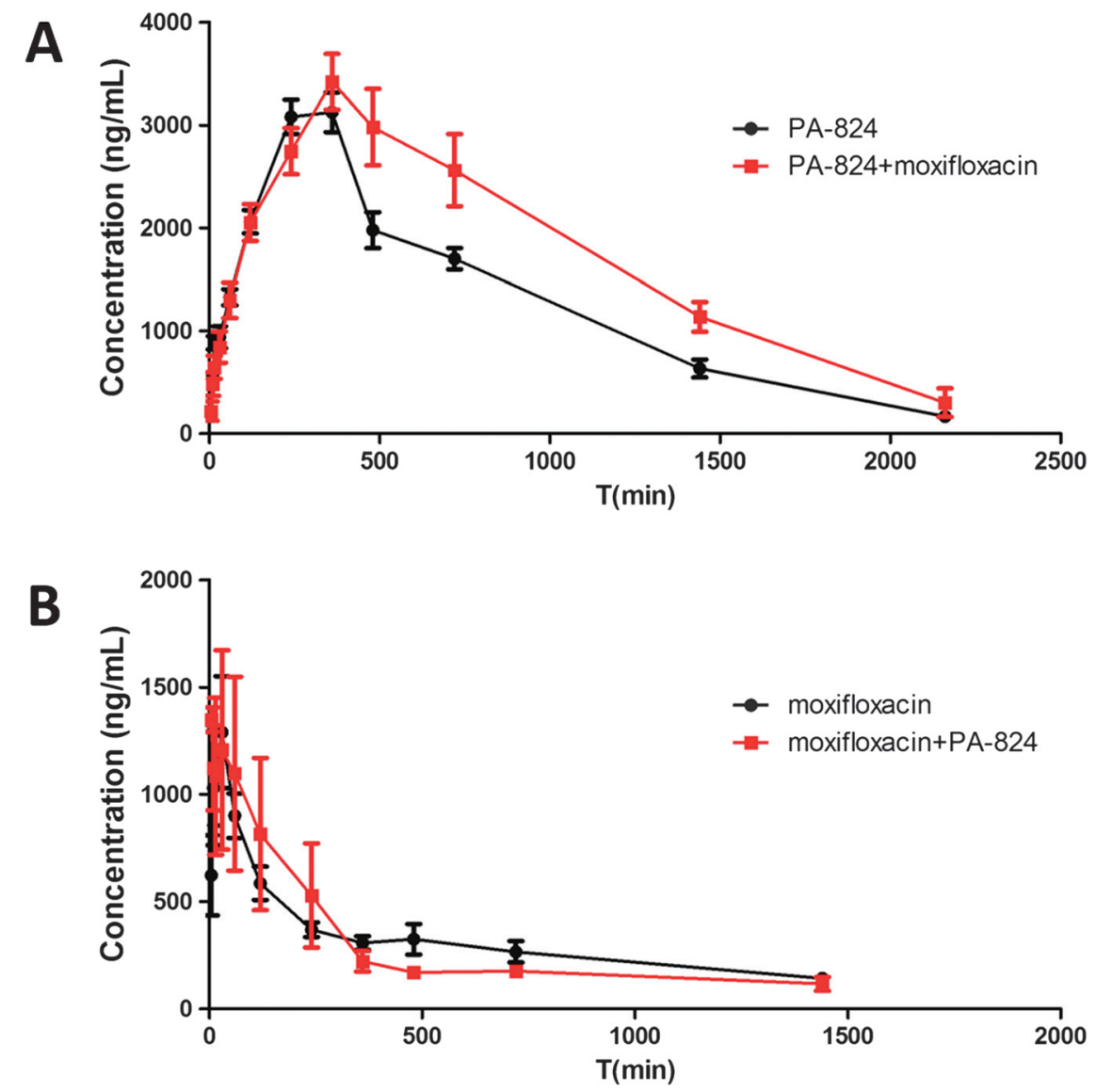

Figure 3. Mean plasma concentration-time curves (mean $\pm \mathrm{SD}, n=8)$ of PA- 824 after oral administration of PA- $824(20 \mathrm{mg} / \mathrm{kg})$ and combined administration of PA-824 (20 mg/kg) and moxifloxacin $(40 \mathrm{mg} / \mathrm{kg})(\mathrm{A})$. Mean plasma concentration-time curves (mean $\pm \mathrm{SD}, n=8)$ of moxifloxacin after oral administration of moxifloxacin $(40 \mathrm{mg} / \mathrm{kg}$ ) and combined administration of moxifloxacin (40 mg/kg) and PA-824 (20 mg/kg) (B) 
Table 4. Pharmacokinetic parameters of PA-824 and moxifloxacin from the single group and the combined group in rats after oral administration

\begin{tabular}{|c|c|c|c|c|}
\hline \multirow[t]{2}{*}{ Parameter } & \multicolumn{2}{|c|}{ Administration of PA-824 $(n=8)$} & \multicolumn{2}{|c|}{ Administration of moxifloxacin $(n=8)$} \\
\hline & Single group & Combined group & Single group & Combined group \\
\hline $\mathrm{AUC}_{(0-t)}(\mathrm{mg} / \mathrm{L} \cdot \min )$ & $2714.5 \pm 1177.6$ & $3706.3 \pm 1150.5$ & $455.3 \pm 111.6$ & $412.4 \pm 201.9$ \\
\hline $\operatorname{AUC}_{(0-\infty)}(\mathrm{mg} / \mathrm{L} \cdot \mathrm{min})$ & $2858.4 \pm 894.6$ & $4119.4 \pm 1602.6$ & $782.4 \pm 5731.3$ & $743.7 \pm 409.3$ \\
\hline$t_{\max }(\min )$ & $270.4 \pm 84.9$ & $375.4 \pm 76.9$ & $37.5 \pm 18.4$ & $22.5 \pm 8.7$ \\
\hline$t_{1 / 2}(\min )$ & $457.3 \pm 110.6$ & $536.1 \pm 213.7$ & $870.6 \pm 464.2$ & $1521.4 \pm 565.6$ \\
\hline $\mathrm{CLz} / \mathrm{F}(\mathrm{L} / \mathrm{min} / \mathrm{kg})$ & $0.007 \pm 0.001$ & $0.005 \pm 0.002$ & $0.066 \pm 0.025$ & $0.068 \pm 0.038$ \\
\hline$C_{\max }(\mathrm{ng} / \mathrm{mL})$ & $3330.4 \pm 441.3$ & $3542.7 \pm 799.3$ & $1411.8 \pm 533.1$ & $1216.2 \pm 622.6$ \\
\hline
\end{tabular}

\section{Conclusion}

In summary, we used an LC-MS/MS method for the simultaneous determination of PA-824 and moxifloxacin in rat plasma. We studied the differences of pharmacokinetic parameters between alone and coadministration of the two drugs, and explored whether there are DDI between them, providing data and theoretical basis for clinical trial.

Acknowledgements. This research was supported by grants from the Shaanxi Collaborative Innovation Project (2014XT-26).

\section{References}

1. Mustafa, A. S. J. Postgrad. Med. 2003, 49, 271-272.

2. Dye, C.; Scheele, S.; Dolin, P.; Pathania, V.; Raviglione, M. C. JAMA, J. Am. Med. Assoc. 2015, 282, 1189-1210.

3. Dye, C.; Scheele, S.; Dolin, P.; Pathania, V.; Raviglione, M. C. JAMA, J. Am. Med. Assoc. 1999, 282, 686.
4. Duncan, K. Tuberculosis 2003, 83, 201-207.

5. Lalloo, Umesh G.; Ambaram, A. Curr. HIV/AIDS Rep. 2010, 7 , 143-151.

6. Lenaerts, A. J. Antimicrob. Agents Chemother. 2005, 2294-2301.

7. AH, D. Antimicrob. Agents Chemother. 2012, 56, 3027-3031.

8. Gardner, C. A.; Acharya, T.; Pablos-Méndez, A. Clin. Chest Med. 2005, 26 341-347.

9. Wang, L.; Xu, Y.; Liang, L.; Diao, C.; Liu, X.; Zhang, J.; Zhang, S. J. Pharm. Biomed. Anal. 2014, 97, 1-8.

10. Wang, L.; Ma, Y.; Duan, H.; Yao, J.; Liang, L.; Zhang, R.; Zhou, X.; Liu, X.; Wang, Q.; Zhang, S. J. Chromatogr. B: Anal. Technol. Biomed. Life Sci. 2015, 1006, 194-200.

11. Lee, S. J.; Desta, K. T.; Eum, S. Y.; Dartois, V.; Cho, S. N.; Bae, D. W.; Shin, S. C. J. Chromatogr. B: Anal. Technol. Biomed. Life Sci. 2015, 138, 1009-1010

12. Vu, D. H.; Koster, R. A.; Alffenaar, J. W; Brouwers, J. R.; Uges, D. R. J. Chromatogr. B: Anal. Technol. Biomed. Life Sci. 2011, 879, 1063-1070.

13. Vishwanathan, K.; Bartlett, M. G.; Stewart, J. T. J. Pharm. Biomed. Anal. 2002, 30, 961-968.

14. Pranger, A. D.; Alffenaar, J. W.; Wessels, A. M.; Greijdanus, B.; Uges, D. R. J. Anal. Toxicol. 2010, 34, 135-141.

15. U. D. O. Health, F. Human Services, Federal Register 2001, 66 , 206-207. 